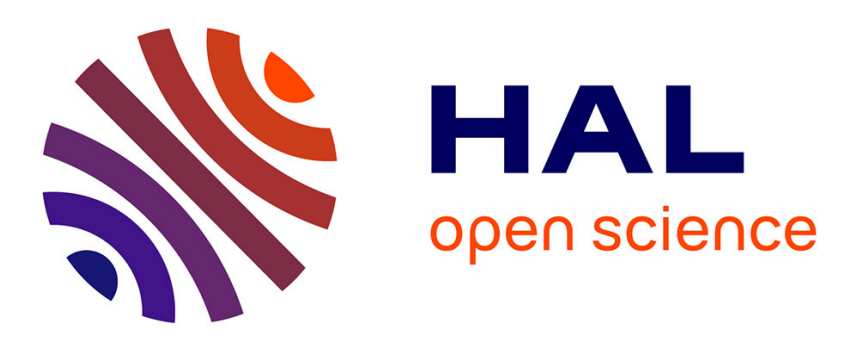

\title{
Modeling Magnetic Near-Field Injection at Silicon Die Level
}

Alexandre Boyer, Bertrand Vrignon, Manuel Cavarroc

\section{To cite this version:}

Alexandre Boyer, Bertrand Vrignon, Manuel Cavarroc. Modeling Magnetic Near-Field Injection at Silicon Die Level. IEEE Transactions on Electromagnetic Compatibility, 2015, 58 (1), pp. 257-269. 10.1109/TEMC.2015.2486041 . hal-01225324

\section{HAL Id: hal-01225324 \\ https://hal.science/hal-01225324}

Submitted on 6 Nov 2015

HAL is a multi-disciplinary open access archive for the deposit and dissemination of scientific research documents, whether they are published or not. The documents may come from teaching and research institutions in France or abroad, or from public or private research centers.
L'archive ouverte pluridisciplinaire HAL, est destinée au dépôt et à la diffusion de documents scientifiques de niveau recherche, publiés ou non, émanant des établissements d'enseignement et de recherche français ou étrangers, des laboratoires publics ou privés. 


\title{
Modeling Magnetic Near-Field Injection at Silicon Die Level
}

\author{
A. Boyer, B. Vrignon, M. Cavarroc
}

\begin{abstract}
Near-field injection at silicon die level is a promising application for various area such as the analyses of integrated circuit (IC) susceptibility to electromagnetic interferences and security for cryptographic applications. This paper presents a first attempt to simulate the voltage induced on integrated circuit interconnects by a magnetic field probe. The validation of the simulation results is based on near-field injection performed on a test chip containing various types of interconnects and on-chip voltage sensors.
\end{abstract}

Index Terms-near-field injection; integrated circuit; susceptibility; electromagnetic attack; modeling; on-chip sensor.

\section{INTRODUCTION}

Near-field scan is a well-established method for the diagnosis of EMC problems at printed circuit board (PCB) and integrated circuit (IC) package levels. It consists in measuring the local electric or magnetic fields created above PCB traces and ICs for a root-cause analysis of emission issues. The method can be reversed to apply local electromagnetic disturbances and is known as near-field injection. A near-field probe is placed in the vicinity of an electronic device and excited by a disturbance signal in order to induce a local intense electric or magnetic field. The coupling of the field may induce enough large voltage fluctuations across the device to trigger failures. In [1] or [2], near-field injection is used to produce susceptibility mapping over PCB and identify sensitive devices or disturbance coupling paths. In [3], the method is extended to characterize susceptibility of ICs by local coupling on IC package up to 6 GHz. The susceptibility to RF disturbances of various circuits were tested based on this method: digital circuits [4], analog-to-digital converter [5], oscillators, PLL and radio receivers [6]. The method was also proposed to investigate the susceptibility of ICs to indirect electrostatic discharge events [7] [8] [9].

Recently, the research community on secure ICs has shown an interest in near-field injection at IC level as low cost,

Manuscript received July *, 2015. This work was supported in part by the French National Research Agency under Project E-Mata-Hari ANR-12-INSE-005-03.

A. Boyer and M. Cavarroc are with LAAS-CNRS, 7 avenue du colonel Roche, F-31077 Toulouse Cedex 4, France (e-mail: alexandre.boyer@laas.fr, manuel.cavarroc@laas.fr).

B. Vrignon is with Freescale Semiconductor, Avenue du general Eisenhower, $31100 \quad$ Toulouse, France (e-mail: Bertrand.vrignon@freescale.com). localized and efficient fault injection method. Experimental results presented in [10] and [11] show that cryptographic circuit operation can be disturbed by electromagnetic (EM) fault attacks produced by a miniature injection probe, resulting in serious threat for systems embedding various types of cryptographic algorithms. The sudden power supply voltage drops, ground bounces and clock glitches induced by the injection probe are able to disturb the normal operation of several building blocks of encryption circuits, such as clock generator disturbance [12], bias increase of random number generator [13], timing constraint violation, bit set and reset in digital flip-flop [11], bit flip in SRAM cells [10]. All these failures can be exploited to extract confidential data such as encryption keys. Besides, local attacks at any point of the die may bypass countermeasures intended to detect fault injection, as shown in [14].

The development of near-field injection at silicon die level is certainly very promising for IC susceptibility to electromagnetic interferences (EMI) and security analyses. The success of this method relies on injection localized on the smallest possible area of the circuit and thus on design of miniature injection probes. But it also relies on accurate models of the physical interaction between the injection probe and the die under test for several reasons: clarifying the nature of the coupling, predicting performances of injection probes, estimating the sensitivity of an circuit building blocks to EMI or the threat of EM attacks, validating by simulation countermeasures against EM fault injection. Some papers such as [15] and [16] report modeling methods of near-field injection on PCB lines or package interconnects. However, the modeling of near-field injection directly at die level has not been investigated and presents some distinctive features because of the multilayer nature of IC internal structure. Compared to the case of injection on PCB traces, the computation of the incoming electric and magnetic fields produced by the injection probe is more complex since it requires a rigorous determination of the Green's functions. Moreover, because of the non homogeneous medium and the presence of lossy materials, propagation along IC interconnects designed on typical silicon substrate is seldom in quasi-TEM mode but rather in slow-wave mode [17]. This behavior must be accounted for to predict voltage fluctuations induced by near-field injection.

The objective of this paper is to develop a modeling method to predict the voltage induced along IC interconnects by near-field injection probes. Previous papers such as [18] and 
[19] have shown some limits of near-field injection based on electric field probes. Therefore the paper is focused only on the modeling of magnetic field injection. The validation of the model relies on a test chip which embeds various interconnect structures terminated by on-chip voltage sensors (OCS) dedicated to the measurement of voltage induced by near-field injection. The paper is organized as follows: Section II presents the expected characteristics of near-field injection probe and their electrical modeling. Section III deals with the modeling flow to predict the $\mathrm{H}$ field coupling on IC interconnect. In particular, this part presents the derivation of analytical expressions of magnetic field Green's functions for a general multilayer environment. These expressions are adapted to any types of $\mathrm{H}$ field probes in arbitrary orientation. Section IV describes the near-field injection scanner, the test chip and the principle of the OCS. Section V presents experimental results of near-field injection on the test chip, which are compared with simulation results in Section VI.

\section{NEAR-FIELD INJECTION PROBE}

\section{A. Characteristics of near-field injection probe}

Near-field probes are miniature wideband antennas usually dedicated to sense either electric or magnetic fields in the vicinity of electronic components, printed circuit boards or antennas. They are usually based on small loops for magnetic field probe and opened tips for electric field probe [20]. If they are excited by an electrical signal, they produce strong electric or magnetic fields in their near-field region, which can couple on a device under test (DUT) to produce important voltage fluctuations locally. Near-field injection relies on probe with a good resolution and a sufficient efficiency to induce a perceptible effect on the DUT. Compared to sensing probes, injection probes have an additional constraint: the maximum current to prevent an excessive heating. The maximum current can be evaluated according to the Onderdonk equation, which relates the fusing current, the wire section, the excitation current and duration [21].

In this study, a handmade tangential $\mathrm{H}$ field probe is tested. A small loop probe with $0.6 \mathrm{~mm}$ diameter is built at the end of a semi-rigid coaxial cable MIL-C-17/151. Its construction is detailed in Fig. 1. In order to improve the probe efficiency, the diameter of the loop or the number of turns should be increased but at the price of a degraded resolution and a larger self inductance. Another alternative to increase the magnetic field in the vicinity of the probe consists in placing a magnetic core inside the coil. However, as the magnetic flux enhancement is related to the ratio coil length on core radius, this is not a practical solution to build compact tangential $\mathrm{H}$ field probe.

\section{B. Near-field probe modeling}

The magnetic field produced by the $\mathrm{H}$ field probe is proportional to the coil excitation current. An accurate electrical model of the probe is required to predict the excitation current. The probe is formed by a length of coaxial cable terminated by a miniature coil. The coaxial cable can be modeled as transmission line while the coil can be represented by a lumped electrical model up to several $\mathrm{GHz}$ due to its small size. The coil is inductive predominantly. An excessive inductance limits the excitation current at high frequency. For a circular loop, the inductance L can be estimated using (1) [22], while models given by Lundin formula (2) may be used for multiturn coil [23].

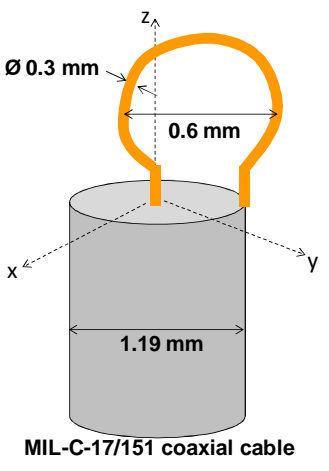

(a)

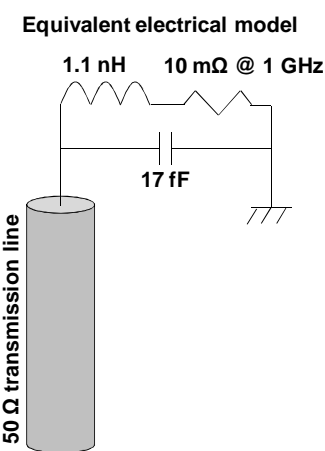

(b)

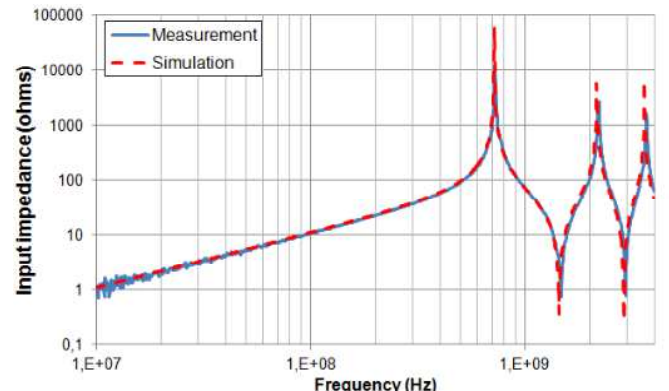

Frequency $(\mathrm{Hz})$

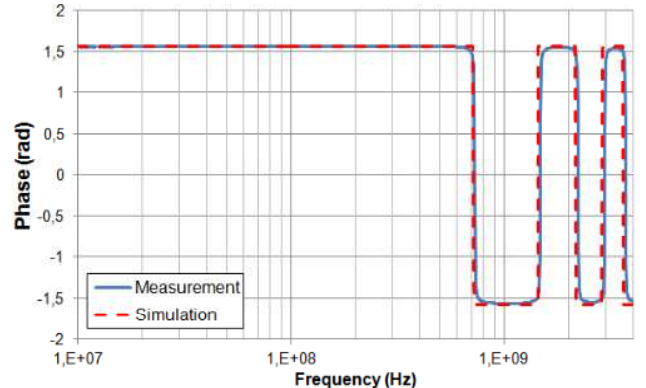

(c)
Fig. 1. Magnetic field probe: (a) Construction details, (b) Electrical modeling, (c) Comparison between measured and simulated input impedance

$$
\begin{aligned}
& L_{1}=\mu_{0} r_{l}\left(\ln \left(\frac{8 r_{l}}{r_{w}}\right)-2\right) \\
& L_{N}=\mu_{0} N^{2} \frac{2 \pi r_{l}^{2}}{l}\left(F_{1}\left(\frac{4 r_{l}^{2}}{l^{2}}\right)-\frac{8}{3 \pi} \frac{r_{l}}{l}\right)
\end{aligned}
$$

with $\mathrm{N}$ the number of turns, 1 the coil length, $\mathrm{r}_{1}$ and $\mathrm{r}_{\mathrm{w}}$ the loop and wire radius. The coefficient $\mathrm{F}_{1}$ is given by (3)

$$
F_{1}(x)=\frac{1+0.38 x+0.017 x^{2}}{1+0.26 x}
$$

The coil is also affected by stray capacitors $\mathrm{C}$ which can be computed according to (4) [22] for a circular loop, and to the Medhurst model [24] for a $\mathrm{N}$ turn coil (5). 


$$
\begin{aligned}
& C_{1}=\frac{4 \varepsilon_{0} r_{l}}{\ln \left(\frac{8 r_{l}}{r_{w}}\right)-2} \\
& C_{N}=\frac{4 \varepsilon_{0}}{\pi} l\left(1+0.71 \frac{2\left(r_{l}+r_{w}\right)}{l}+2.395\left(\frac{2\left(r_{l}+r_{w}\right)}{l}\right)^{1.5}\right)
\end{aligned}
$$

Resistors due to ohmic and radiation losses may be included but their influence remains negligible in practice. Fig. 1 presents the equivalent electrical model of the magnetic field probe. With (1) and (4), the inductance and capacitance of our probe are estimated to $1.1 \mathrm{nH}$ and $17 \mathrm{fF}$. These values are confirmed by full-wave electromagnetic simulations. A comparison between the measured and simulated input impedance of the probe is presented in Fig. 1-c, showing a quite good agreement. For a circular conductor and considering skin effect, the ohmic losses of the probe are modeled by a $10 \mathrm{~m} \Omega$ resistor at $1 \mathrm{GHz}$. Due to the small size of the probe, stray capacitance and loss resistance have a negligible influence up to several gigahertz actually. Moreover, the existence of parasitic common-mode current can also be neglected. For a given probe excitation, the excitation current amplitude along the loop remains constant.

\section{MODELING OF NEAR-FIELD INJECTION ON INTEGRATED CIRCUIT INTERCONNECTS}

\section{A. Modeling of near-field injection on a transmission line}

The coupling of electromagnetic fields produced by near-field probes on PCB traces or package interconnects have been addressed in some publications such as [15] and [16]. It consists in predicting the response of a quasi-TEM guided transmission to non uniform electric or magnetic field which decays rapidly with the distance. Solutions are provided by coupling equations such as those given by the Taylor model [25]. The coupling of the external electric and magnetic fields results in either distributed voltage or current sources. The terminal voltages and currents are determined by the resolution of the Telegrapher's equations, for example according to the BLT equations formalism [26], or by an electrical simulator such as SPICE.

The evolution of the electric or magnetic field emitted by the injection probe according to the distance must be computed accurately. For $\mathrm{H}$ field probe, a simple approach consists in using the magnetic dipole approximation. However, if probe dimensions are not negligible compared to the separation distance with the device under test or scan altitude, the probe emission model is not accurate enough. The dyadic Green's function of the surrounding environment may be established in order to compute the contribution of every part of the injection probe to the emitted magnetic field. For injection on PCB, traces are routed on insulating material with the possible presence of a highly conductive ground plane. The potential vector Green's function due to an elementary current filament is easily derived by considering free space conditions and image theory in case of the presence of a ground plane. The methodology to predict the coupling of a $\mathrm{H}$ field probe and a transmission line is summed up in Fig. 2.

This method can be extended to the coupling of $\mathrm{H}$ field on IC interconnects but two difficulties arise. Firstly, due to the resistivity of the semiconducting substrate, the near-field probe induces complicated eddy currents within the substrate which may affect the distribution of the magnetic field. Its rigorous determination requires the extraction of the magnetic field dyadic Green's function in this multilayer planar environment. Secondly, propagation modes in IC interconnects designed on a lossy silicon substrate are different from the usual quasi-TEM mode in lines designed on insulating substrates and extremely dispersive with frequency. The following parts present the methods used to model both essential aspects for the prediction of the magnetic field coupling on IC interconnects.

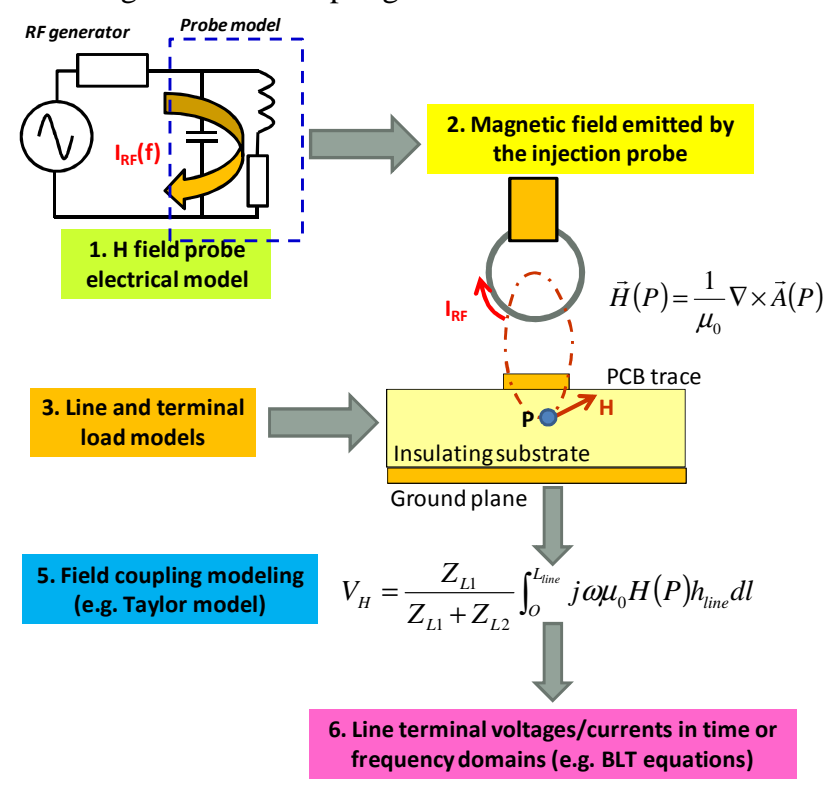

Fig. 2. Typical flow to model magnetic field injection on transmission line

\section{B. Modeling of the propagation along IC interconnects}

IC interconnects are designed on conductive layers isolated by insulating layer from the semiconducting bulk substrate. A simple geometrical model to analyze the signal transmission along IC interconnects is the Metal-Insulator Semiconductor (MIS) microstrip line described in Fig. 3.

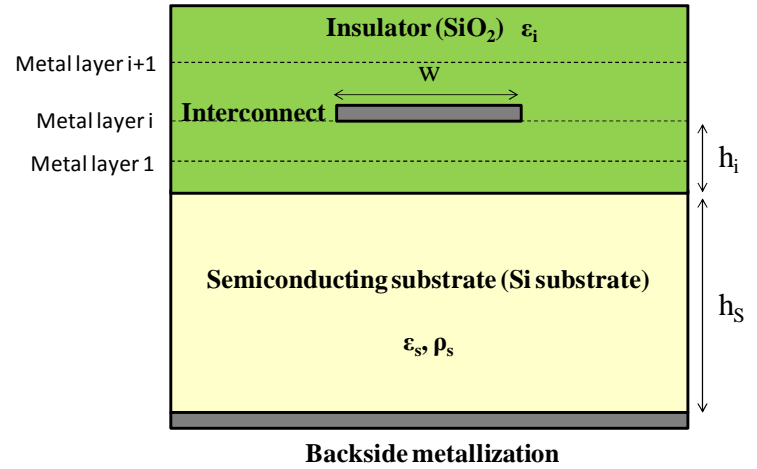

Fig. 3. Metal-Insulator-Semiconductor (MIS) microstrip line 
The back metallization is formed either directly on the back side of the chip or associated to the cavity of the circuit package. The structure is supposed linear: the influence of the voltage bias on the propagation characteristics is neglected.

The analysis of the wave propagation along a MIS microstrip line has been dealt in numerous papers based on various frequency domain numerical methods, such as finite element method [27] or spectral-domain method [28]. Closed-form expressions of the line parameters have also been derived from complex image method [29] or theoretical analysis of a parallel-plate waveguide [17]. Compared to numerical methods or approximations based on complex image method, the theoretical analysis of a parallel-plate waveguide provides a physical understanding of the existing propagation modes and an accurate evaluation of the line characteristics.

When the substrate resistivity is high, the substrate can be considered as an insulator and the dielectric quasi-TEM mode dominates. However, silicon substrate is not a good insulator since its resistivity ranges between 0.01 and $10 \Omega . \mathrm{cm}$. The rigorous analysis presented in [17] shows that three propagation modes may exist depending on the substrate resistivity and frequency:

-- At low frequency, only the slow-wave mode propagates. The creation of a polarization at the $\mathrm{Si}-\mathrm{SiO}_{2}$ interface increases the electrical permittivity due to the Maxwell-Wagner mechanism and slows down the propagation velocity. The magnetic field penetrates the substrate, contrary to the electric field which is contained in the insulator region.

-- At high frequency, the substrate acts as an imperfect electric wall with a large skin effect, resulting in a skin effect propagation mode.

-- Depending on the substrate resistivity, quasi-TEM mode may exist in-between.

In the first two modes, the propagation characteristics are dispersive with frequency. An equivalent circuit representation of a MIS microstrip line is derived from the parallel-plate waveguide model, as shown in Fig. 4. Closed-form expressions of RLCG parameters can be found in [30].

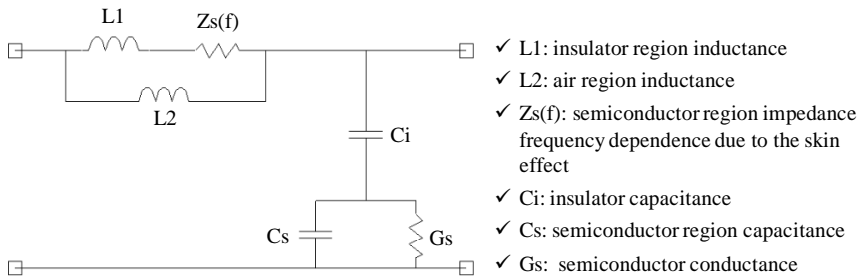

Fig. 4. Equivalent circuit representation of a MIS microstrip line

\section{Analytical expressions for magnetic field dyadic Green's functions}

Fig. 5 describes the geometry of the general multilayer substrate, the position of the probe and the coordinate system. An arbitrary shaped and oriented $\mathrm{H}$-field probe excited by a known current is placed just above the die at a separation distance called scan altitude $\mathrm{h}_{\text {scan }}$. The die is considered as an infinitely large planar multilayered medium. The coupling between an interconnect routed on the die and the $\mathrm{H}$ field probe is related to the $\mathrm{H}$ field produced by the probe in the different layers of the die. It should be emphasized that the injection probe is placed as close as possible to the die and typical die thickness is only some hundreds of $\mu \mathrm{m}$ so the magnetic field is computed at very short distance to the probe.

The $\mathrm{H}$ field probe can be broken down into several elementary filaments crossed by a known current density noted $\mathrm{J}$. The $\mathrm{H}$ field produced by the probe is the sum of the contribution of each current filament centered in a source point noted S. The current filaments should be short compared to the wavelength and smaller than the separation distance $h_{\text {scan }}$ to predict the emitted magnetic field accurately. An adequate number of current filaments may be selected iteratively in order to ensure a convergence of the magnetic field computation. In the next part, the injection probe is divided in 12 filaments. A good convergence of the magnetic field is ensured up to $3 \mathrm{GHz}$ and for scan altitude of $150 \mu \mathrm{m}$.

The layers are defined by the superscript $i j$, where $i=1$ for any layers below $S$ and 2 for the layer above $S$, and $j$ indicates the number of the lower or upper layer of the observation point. The source point $S$ is always at the interface between layers 12 and 21. The magnetic field at the $\mathrm{P}$ is computed according to (6), where $\overline{\overline{G_{H}^{1 N}}}$ is the magnetic field dyadic Green's function for an observation point in layer $1 \mathrm{~N}$.

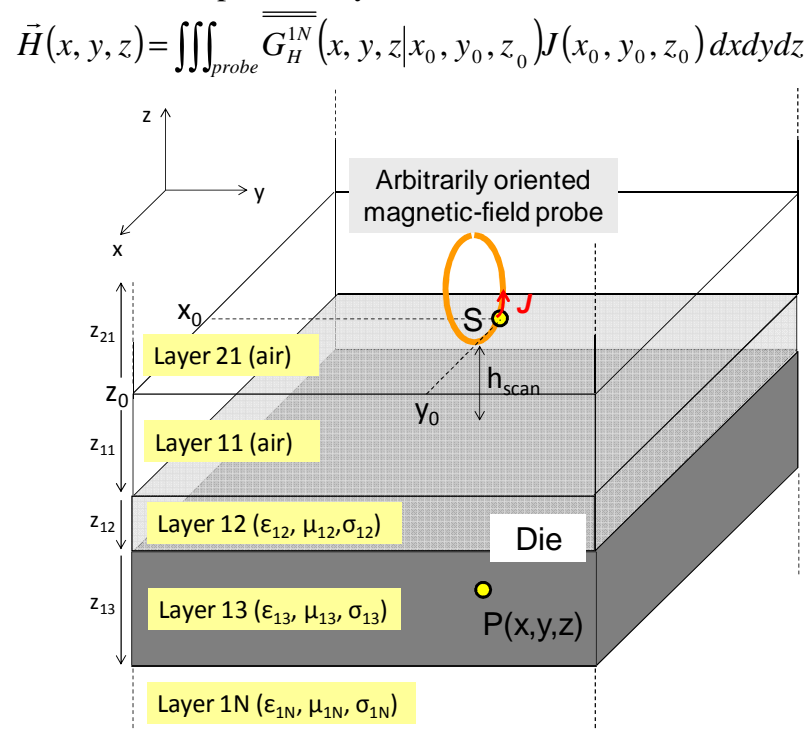

Fig. 5. Geometry of the multilayer substrate and position of the injection probe

Traditionally, the Green's function in spatial domain is represented by the Sommerfeld integrals extracted from the Maxwell equations and boundary conditions at each layer interface. The transform in spectral domain has been widely used in microwave circuits applications in order to obtain closed-form expressions of Green's function in a two layer problem [31] [32]. For multilayer substrate, analytical expressions of dyadic Green's function can be derived by using iterative algorithms to take into account the effect of other layers [33] [34]. The Green's function in spatial domain is then determined by a numerical integration of the Sommerfeld 
integral expressed in spectral domain.

However, the slowly decaying and oscillatory behavior of the Green's function, especially at large distance, reduces the efficiency of the computation. Numerous papers describe methods to extract closed-form expressions of Green's function in spatial domain, such as Complex image theory or Discrete Complex Image Method (DCIM) [29] [34] [35]. In spite of a significant reduction of the computation time compared to the numerical integration of the Sommerfeld integral, the accuracy of these methods may be compromised if other wave contributions than spherical waves are accounted for [36].

Considering near-field injection at die level, only the field in close proximity to the probe needs to be computed so the Green's function decays rapidly without large oscillations. The numerical integration of the Sommerfeld integral remain quite efficient, without the risk of inaccuracies due to approximation in spherical waves. In the following parts, the approach described in [33] to compute analytical expressions of electric and magnetic vector potential Green's functions in general multilayer substrates is reused in order to compute the magnetic field dyadic Green's function. The expressions are derived in spectral domain first, and then in spatial domain for an arbitrary oriented elementary current filament.

\section{1) Spectral-domain expressions for the $H$-field dyadic}

Green's function in a multilayered substrate

An elementary filament excited by a sinusoidal current is considered at the interface between layers 11 and 21 . In the

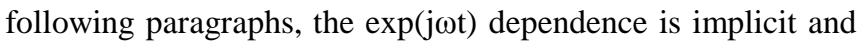
only the expressions of the magnetic field in the lower layers are investigated. The relations between spatial and spectral domain representation of the magnetic field is ensured by a $2 \mathrm{D}$ Fourier transform in the xy plane, as given by (7) and (8) where subscript $\tilde{H}$ denotes the spectral domain representation. The field is decomposed into a superposition of an infinite number of plane waves propagating in $\mathrm{x}$ and $\mathrm{y}$ directions with wave numbers $\mathrm{k}_{\mathrm{x}}$ and $\mathrm{k}_{\mathrm{y}}$.

$$
\begin{aligned}
& \tilde{H}_{x, y, z}\left(k_{x}, k_{y}, z\right)=\iint_{-\infty}^{+\infty} H_{x, y, z}(x, y, z) e^{-j k_{x} x} e^{-j k_{y} y} d x d y \\
& H_{x, y, z}(x, y, z)=\frac{1}{4 \pi^{2}} \iint_{-\infty}^{+\infty} \tilde{H}_{x, y, z}\left(k_{x}, k_{y}, z\right) e^{j k_{x} x} e^{j k_{y} y} d k_{x} d k_{y}
\end{aligned}
$$

In [33], analytical expressions are proposed for the electric and magnetic potential vectors $\mathrm{A}$ and $\mathrm{F}$ Green's function produced by an elementary current source oriented along $\mathrm{x}$ or $\mathrm{y}$ axis. The three components of the $\mathrm{H}$ field dyadic Green's function induced by $\mathrm{x}$ or $\mathrm{y}$ axis oriented current source can be computed according to (9)-(11). The subscript $H_{x} J_{x}$ under $G$ indicates the $\mathrm{H}$ field component produced by the $\mathrm{x}$ oriented current and $\mathrm{k}$ is the wave number in the considered layer.

$$
\begin{aligned}
& \tilde{G}_{H x J x}=j k_{y} \tilde{G}_{A z J x}+\frac{k_{x}}{\omega \mu} \frac{d \tilde{G}_{F z J x}}{d z} \\
& \tilde{G}_{H y J x}=-j k_{x} \tilde{G}_{A z J x}+\frac{k_{y}}{\omega \mu} \frac{d \tilde{G}_{F z J x}}{d z}
\end{aligned}
$$

$$
\tilde{G}_{H z J x}=\frac{1}{j \omega \mu}\left(\frac{d^{2} \tilde{G}_{F z J x}}{d z^{2}}+k^{2} \tilde{G}_{F z J x}\right)
$$

The component of the dyadic due to a $\mathrm{z}$ axis oriented current source oriented can be extracted from the reciprocity theorem and geometrical symmetry [32] (12) (13). The following paragraphs detail the derivation of the terms $\mathrm{H}_{\mathrm{x}} \mathrm{J}_{\mathrm{x}}, \mathrm{H}_{\mathrm{y}} \mathrm{J}_{\mathrm{x}}, \mathrm{H}_{\mathrm{z}} \mathrm{J}_{\mathrm{x}}$ and $\mathrm{H}_{\mathrm{x}} \mathrm{J}_{\mathrm{z}}$, since they are sufficient to deduce the other terms of the dyadic.

$\tilde{G}_{H x J z}\left(k_{x}, k_{y}, z / x_{0}, y_{0}, z_{0}\right)=\tilde{G}_{H z J x}\left(k_{x}, k_{y}, z_{0} / x_{0}, y_{0}, z\right)$
$\tilde{G}_{H x J z}\left(-k_{y}, k_{x}, z / x_{0}, y_{0}, z_{0}\right)=-\tilde{G}_{H y J x}\left(k_{x}, k_{y}, z_{0} / x_{0}, y_{0}, z\right)$

If the observation point lies in the same layer than the source, the dyadic components produced by a current source oriented along $\mathrm{x}$ axis can be computed according to (14)-(17). The terms $\mathrm{a}_{11}, \mathrm{f}_{11}$ and $\mathrm{f}_{21}$ are the contributions of magnetic and electric potential vectors. They are given by (20)-(22).

$$
\begin{aligned}
& \tilde{G}_{H x J x}^{11}=\frac{k_{x} u_{11}}{\omega \mu_{11}}\left(e^{u_{11} z}-\Gamma_{F 11} e^{-u_{11} z}\right) f_{11}+j k_{y}\left(e^{u_{11} z}+\Gamma_{A 11} e^{-u_{11} z}\right) a_{11} \\
& \tilde{G}_{H y J x}^{11}=\frac{k_{y} u_{11}}{\omega \mu_{11}}\left(e^{u_{11} z}-\Gamma_{F 11} e^{-u_{11} z}\right) f_{11}-j k_{x}\left(e^{u_{11} z}+\Gamma_{A 11} e^{-u_{11} z}\right) a_{11} \\
& \tilde{G}_{H z J x}^{11}=\frac{\left(k_{x}^{2}+k_{y}^{2}\right)}{j \omega \mu_{11}}\left(e^{u_{11} z}+\Gamma_{F 11} e^{-u_{11} z}\right) f_{11} \\
& \tilde{G}_{H x J z}^{11}=\tilde{G}_{H z J x}^{21}=\frac{\left(k_{x}^{2}+k_{y}^{2}\right)}{j \omega \mu_{11}}\left(e^{-u_{z 1} z}+\Gamma_{F 21} e^{u_{21} z}\right) f_{21}
\end{aligned}
$$

with:

$u_{i j}=\left\{\begin{array}{l}j \sqrt{k_{i j}^{2}-k_{x}^{2}-k_{y}^{2}} \text { if } k_{x}^{2}+k_{y}^{2} \leq k_{i j}^{2} \\ \sqrt{k_{x}^{2}+k_{y}^{2}-k_{i j}^{2}} \text { if } k_{x}^{2}+k_{y}^{2}>k_{i j}^{2}\end{array}\right.$

and $k_{i j}=k_{0} \sqrt{\varepsilon_{r i j}}$

$a_{11}=\frac{-k_{x} \varepsilon_{11} u_{21}\left(1-\Gamma_{A 21}\right)}{j\left(k_{x}^{2}+k_{y}^{2}\right) T_{A}}$

$f_{11}=\frac{k_{y} \omega \mu_{11}\left(1+\Gamma_{F 21}\right)}{\left(k_{x}^{2}+k_{y}^{2}\right) T_{F}}$

$f_{21}=\frac{k_{y} \omega \mu_{21}\left(1+\Gamma_{F 11}\right)}{\left(k_{x}^{2}+k_{y}^{2}\right) T_{F}}$

with:

$T_{A}=\varepsilon_{11} u_{21}\left(1+\Gamma_{A 11}\right)\left(1-\Gamma_{A 21}\right)+\varepsilon_{21} u_{11}\left(1+\Gamma_{A 21}\right)\left(1-\Gamma_{A 11}\right)$

$T_{F}=\mu_{11}\left(1-\Gamma_{F 11}\right)\left(1+\Gamma_{F 21}\right)+\mu_{21}\left(1-\Gamma_{F 21}\right)\left(1+\Gamma_{F 11}\right)$

$\Gamma_{\mathrm{A} 11}$ and $\Gamma_{\mathrm{F} 11}$ are equivalent to reflection coefficients due to the other lower layer interfaces. In [33], an iterative algorithm is proposed to compute the value of the reflection coefficient for each layer, by analogy with a transmission line. The reflection coefficient of the magnetic and electric potential vectors are computed recursively according to (25) and (26).

$\Gamma_{A i j}=e^{-2 u_{i j} D_{i j}} \frac{Z_{A i j}-Z_{A i j+1}^{T}}{Z_{A i j}+Z_{A i j+1}^{T}}$ 
with $Z_{A i j}=-j \frac{u_{i j}}{\varepsilon_{i j}}$ and $Z_{A i j}^{T}=\frac{1-\Gamma_{A i j+1}}{1+\Gamma_{A i j+1}} Z_{A i j+1}$

$\Gamma_{F i j}=e^{-2 u_{i j} D_{i j}} \frac{Y_{F i j}-Y_{F i j+1}^{T}}{Y_{F i j}+Y_{F i j+1}^{T}}$

with $Y_{F i j}=-j \frac{u_{i j}}{\mu_{i j}}$ and $Y_{F i j}^{T}=\frac{1-\Gamma_{F i j+1}}{1+\Gamma_{F i j+1}} Y_{F i j+1}$

Some special boundary conditions are necessary: if the layer ij is infinite, no reflection occurs so $\Gamma_{\mathrm{Aij}}$ and $\Gamma_{\mathrm{Fij}}$ are zeros. If the layer ij is a perfect electrical conductor, $\Gamma_{\mathrm{Aij}}=+1$ and $\Gamma_{\mathrm{Fij}}=-1$ while $\mathrm{Z}_{\mathrm{Aij}}=0$ and $\mathrm{Y}_{\mathrm{Fij}}=\infty$. The dyadic components given by (14)-(17) can be generalized for any lower layers by replacing the terms $a_{11}$ and $f_{11}$ by $a_{1 N}$ and $f_{1 N}$, given by the following iterative process.

$$
a_{1 N}=a_{1 N-1} \prod_{j=2}^{N-1} K_{A 1 j}
$$

$$
\begin{aligned}
& \text { with } K_{A 1 j}=Z_{A 1 j} \frac{2 e^{-u_{1 j} D_{1 j}}}{\left(1+\Gamma_{A 1 j+1}\right)\left(Z_{A 1 j}+\frac{1-\Gamma_{A 1 j+1}}{1+\Gamma_{A 1 j+1}} Z_{A 1 j+1}\right)}, j \geq 2 \\
& f_{1 N}=f_{1 N-1} \prod_{j=2}^{N-1} K_{F 1 j}
\end{aligned}
$$

$$
\text { with } K_{F 1 j}=Y_{F 1 j} \frac{2 e^{-u_{1 j} D_{1 j}}}{\left(1+\Gamma_{F 1 j+1}\right)\left(Y_{F 1 j}+\frac{1-\Gamma_{F 1 j+1}}{1+\Gamma_{F 1 j+1}} Y_{F 1 j+1}\right)}, j \geq 2
$$

2) Spatial-domain expressions for the H-field dyadic Green's function in a multilayered substrate

In order to compute the space domain Green's function of the magnetic field, a 2D inverse Fourier transform must be performed (8). It can be simplified to a 1D Fourier-Hankel transform if the Green's function terms are expressed in a polar coordinate system, with:

$\left\{\begin{array}{l}x=\rho \cos \theta \\ y=\rho \sin \theta\end{array}\right.$ and $\left\{\begin{array}{l}k_{x}=\lambda \cos \alpha \\ k_{y}=\lambda \sin \alpha \Rightarrow k_{x} x+k_{y} y=\lambda \rho \cos (\alpha-\theta)\end{array}\right.$

The different Green's function terms for an observation point in the $\mathrm{N}^{\text {th }}$ lower layer are given by (30)-(33).

$$
\begin{aligned}
& \tilde{G}_{H x J x}^{1 N}=\frac{1}{2}\left(A_{5}^{1 N} \prod_{j=2}^{N} K_{F 1 j}-A_{6}^{1 N} \prod_{j=2}^{N} K_{A 1 j}\right) \sin (2 \alpha) \\
& \tilde{G}_{H y J x}^{1 N}=A_{5}^{1 N} \sin ^{2}(\alpha) \prod_{j=2}^{N} K_{F 1 j}+A_{6}^{1 N} \cos ^{2}(\alpha) \prod_{j=2}^{N} K_{A 1 j} \\
& \tilde{G}_{H z J x}^{1 N}=\lambda \sin (\alpha) A_{4}^{1 N} \prod_{j=2}^{N} K_{F 1 j} \\
& \tilde{G}_{H x J z}^{1 N}=\tilde{G}_{H z J x}^{2 N}=\lambda \sin (\alpha) A_{4}^{2 N} \prod_{j=2}^{N} K_{F 2 j}
\end{aligned}
$$

with:

$$
A_{5}^{1 N}=\frac{u_{1 N}\left(1+\Gamma_{F 21}\right)\left(e^{u_{1 N} z}+\Gamma_{F 1 N} e^{-u_{1 N} z}\right)}{T_{F}}
$$

$$
\begin{aligned}
A_{6}^{1 N} & =\frac{\varepsilon_{11} u_{21}\left(1-\Gamma_{A 21}\right)\left(e^{u_{1 N} z}+\Gamma_{A 1 N} e^{-u_{1 N} z}\right)}{T_{A}} \\
A_{4}^{1 N} & =\frac{\left(1+\Gamma_{F 21}\right)\left(e^{u_{1 N} z}-\Gamma_{F 1 N} e^{-u_{1 N} z}\right)}{j T_{F}} \\
A_{4}^{2 N} & =\frac{\left(1+\Gamma_{F 11}\right)\left(e^{-u_{12} z}+\Gamma_{F 2 N} e^{-u_{2 N} z}\right)}{j T_{F}}
\end{aligned}
$$

According to the previous coordinate conversion, the inverse Fourier transform is expressed in the following form.

$$
H_{x, y, z}(x, y, z)=\frac{1}{4 \pi^{2}} \int_{0}^{+\infty} \int_{0}^{2 \pi} \tilde{H}_{x, y, z}(\lambda, \alpha, z) e^{j \lambda \rho \cos (\alpha-\theta)} d \alpha \lambda d \lambda
$$

Introducing spectral domain Green's function terms given by (30)-(33) in (38), separating the terms dependent on $\lambda$ and $\alpha$, and using properties of Bessel functions, the expressions of the dyadic Green's function terms in spatial domain given by (39)-(42) are obtained. $J_{m}$ are Bessel functions of the first kind of order $\mathrm{m}$.

$$
\begin{aligned}
G_{H x J x}^{1 N}= & -\frac{1}{4 \pi} \sin (2 \theta) \int_{0}^{\infty} \lambda\left(A_{5}^{1 N} \prod_{j=2}^{N} K_{F 1 j}-A_{6}^{1 N} \prod_{j=2}^{N} K_{A 1 j}\right) J_{2}(\lambda \rho) d \lambda \\
G_{H y J x}^{1 N}= & \frac{1}{4 \pi} \int_{0}^{\infty} \lambda\left\{\left(A_{5}^{1 N} \prod_{j=2}^{N} K_{F 1 j}+A_{6}^{1 N} \prod_{j=2}^{N} K_{A 1 j}\right) J_{0}(\lambda \rho)+\right. \\
& \left.\left(A_{5}^{1 N} \prod_{j=2}^{N} K_{F 1 j}-A_{6}^{1 N} \prod_{j=2}^{N} K_{A 1 j}\right) \cos (2 \theta) J_{2}(\lambda \rho)\right\} d \lambda \\
G_{H z J x}^{1 N}= & \frac{j}{2 \pi} \sin \theta \int_{0}^{\infty} \lambda^{2} A_{4}^{1 N} \prod_{j=2}^{N} K_{F 1 j} J_{1}(\lambda \rho) d \lambda \\
G_{H x J z}^{1 N}= & -\frac{j}{2 \pi} \sin \theta \int_{0}^{\infty} \lambda^{2} A_{4}^{2 N} \prod_{j=2}^{N} K_{F 2 j} J_{1}(\lambda \rho) d \lambda
\end{aligned}
$$

The other terms of the dyadic do not require numerical integration. They can be determined according to (43)-(46).

$G_{H x J y}^{1 N}=-G_{H y J x}^{1 N}$

$G_{H y J y}^{1 N}=-G_{H x J x}^{1 N}$

$G_{H z J y}^{1 N}=-\frac{1}{\tan \theta} G_{H z J x}^{1 N}$

$G_{H y J z}^{1 N}=-\frac{1}{\tan \theta} G_{H x J z}^{1 N}$

The Green function converges with $\lambda$, and the sampling on $\lambda$ may be limited to a maximum value such that $e^{-2 \lambda \rho}$ is less than a preassigned value [33]. The sampling step $d \lambda$ is inversely dependent on the lateral distance $\rho$. As near-field injection are local, $\rho$ is small, so the number of steps for the numerical integrations of (41)-(44) remains limited.

\section{3) Validation of the expressions for the H-field dyadic}

Green's function in a multilayered substrate

In order to validate the space-domain expressions of the Green's function of the magnetic field, comparisons of the results with those provided by the commercial 3D electromagnetic simulator FEKO [37] are proposed. Fig. 6 describes the simulated case study, which consists in a elementary current filament placed at $1 \mathrm{~mm}$ above a two layered substrate. Layer thickness, permittivity and conductivity are 
typical of silicon dies. Fig. 7 presents two results: the first graph shows the evolution of the tangential magnetic field Hy produced by the elementary current filament according to the height $\mathrm{z}$ at $1 \mathrm{GHz}$, while the second presents the evolution with frequency of the magnetic field $300 \mu \mathrm{m}$ below the surface of the $\mathrm{SiO} 2$ layer. The simulations have been done for various substrate conductivity, ranging from 0.005 to $10 \Omega . \mathrm{cm}$. Both methods provide similar results which confirm the validity of the expressions of the Green's function. The simulations show clearly the influence of substrate conductivity and frequency on the penetration of the magnetic field within the substrate.

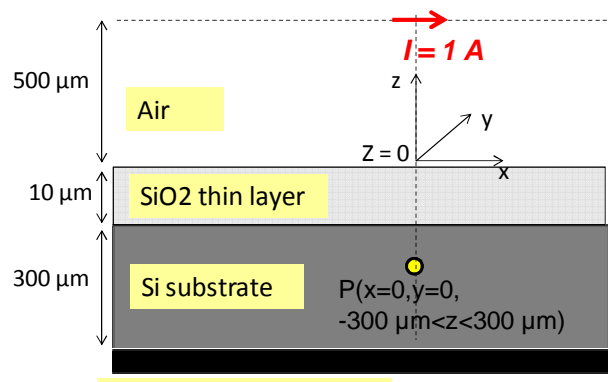

Ideal ground plane

Fig. 6. Description of the validation case
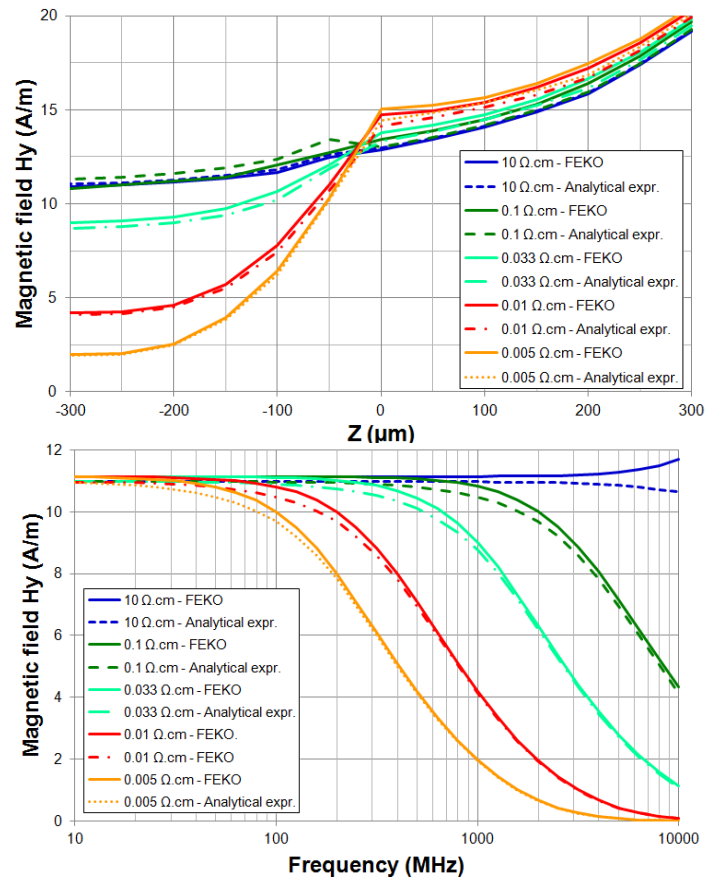

Fig. 7. Evolution of magnetic field vs. distance to current filament at $1 \mathrm{GHz}$ (top) - Evolution of magnetic field vs. frequency at $\mathrm{z}=300 \mu \mathrm{m}$ (bottom)

\section{PRESENTATION OF THE TEST BENCH}

\section{A. Presentation of the test chip}

A test chip has been designed with Freescale ${ }^{\circledR}$ in $0.25 \mu \mathrm{m}$ SMARTMOS 8 technology with 4 metal layers in order to study the near-field injection on basic interconnects and bus structures. Twenty four OCS are disseminated within the test chip to monitor local voltage fluctuations induced by the near-field injection. The test chip is mounted in CQFP64 package with a removable metallic lid in order to place the near-field probe as close as possible to the die surface.

\section{1) Test structures description}

Two test structures are considered in this study, whose characteristics are given in Table I. Fig. 8 shows the dimensions of the chip and the positions of the tested structures. The first structure called Struct1 is detailed in Fig. 9. It contains eight 1 $\mathrm{mm}$ long $50 \Omega$ microstrip lines designed in metal 3 layer, above a reference plane designed in metal 2 layer. The lines are terminated by $50 \Omega$ resistors and an OCS is connected at one end of each line in order to monitor the voltage profile in time domain. The sensors are supplied between $2.5 \mathrm{~V}$ and $0 \mathrm{~V}$. In order to set the line potential at the mid range of the sensor input voltage, the reference plane is biased at $1.25 \mathrm{~V}$ by a bandgap voltage reference $\left(\mathrm{V}_{\mathrm{REF}}\right)$. The eight lines are separated by a variable spacing. The line name depends on the separation to the first one: $0,0.455,5.5,10,30,70,120$ and $320 \mu \mathrm{m}$. For example, the first line is called Line 0 , the second line Line $0.455 \ldots$

The second structure called Struct 2 is described in Fig. 10. It is typical of a digital bus. It contains two $0.5 \mathrm{~mm}$ long lines separated by $0.9 \mu \mathrm{m}$. The lines are driven and loaded by digital buffers. They are routed above a reference plane biased also by the reference $\mathrm{V}_{\mathrm{REF}}$. A shielding in top metal layer is placed above Struct2.

TABLE I. CHARACTERISTICS OF THE TESTED STRUCTURES

\begin{tabular}{|l|l|}
\hline \multicolumn{1}{|c|}{ Name } & Description \\
\hline $\begin{array}{l}\text { Struct1 - Microstrip } \\
\text { line }\end{array}$ & $\begin{array}{l}\text { Eight } 1 \mathrm{~mm} \text { line in M3 loaded by } 50 \Omega \text { resistors at } \\
\text { each end, separated with variable spacing }\end{array}$ \\
\hline $\begin{array}{l}\text { Struct2 - Digital line } \\
\text { with metal shielding }\end{array}$ & $\begin{array}{l}\text { Two } 0.5 \mathrm{~mm} \text { lines in } \mathrm{M} 3 \text { connecting digital } \\
\text { buffers, separated by } 0.9 \mu \mathrm{m}\end{array}$ \\
\hline
\end{tabular}

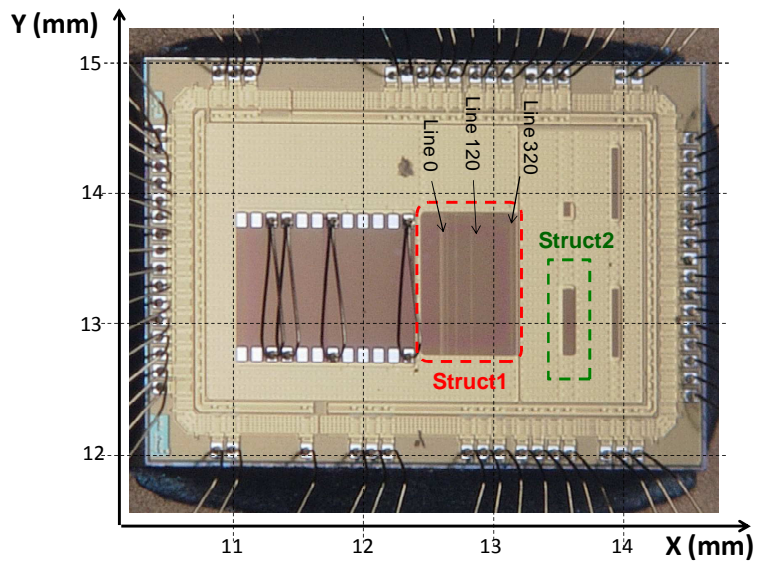

Fig. 8. Test chip and position of the tested structures

2) On-chip sensor

The OCS is able to measure the waveform of voltage bounces across non accessible nodes with a precise time resolution, a large bandwidth and a low intrusivity. The acquisition principle is based on a sequential equivalent-time sampling (Fig. 11), which provides a very large virtual bandwidth in spite of a 
limited sampling rate. Its principle is explained in [38]. In order to prevent noise coupling to sensors, they are supplied by an internal voltage regulator connected to a dedicated power supply and are isolated from the bulk and conductive substrate by a deep $\mathrm{N}$-well. Moreover the OCS is routed with only three metal layers. A complete shielding of the OCS is done with the top level metal layer in order to limit the disturbance of the OCS due to near-field injection. The reader can refer to [39] for more details about the sensor designs and performances.

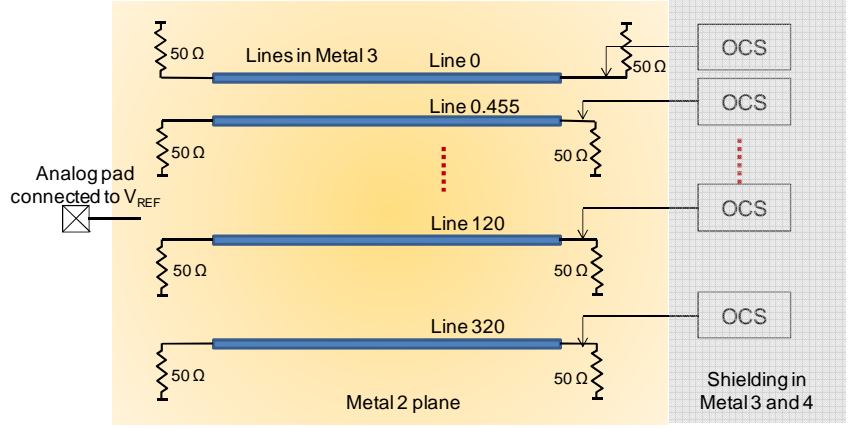

Fig. 9. Schematic of Struct1

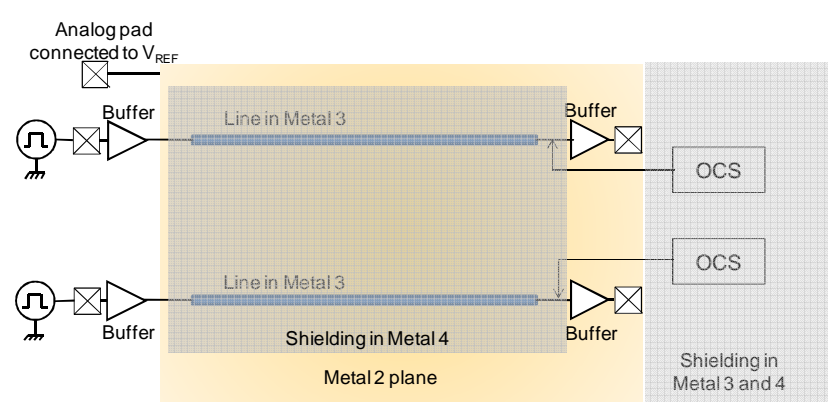

Fig. 10. Schematic of Struct2

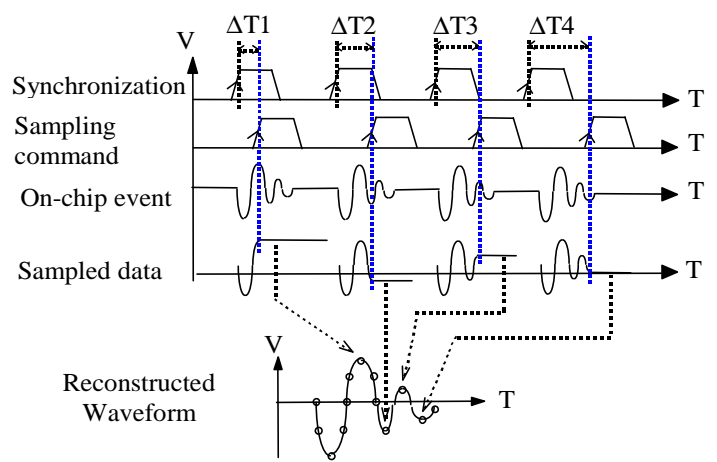

Fig. 11. Principle of signal reconstruction with the on-chip sensor [37]

The sensor is controlled by an external acquisition card which sends sampling clock, delay control commands and converts the analog signals delivered by the sensor. The waveform of a signal can be reconstructed only if it is reproducible and synchronized with the sampling command of the sensor. During near-field injection, the probe excitation source is synchronized with the OCS sampling command.

The characteristics of the OCS depends on the fabrication technology. With a CMOS $0.25 \mu \mathrm{m}$ technology, the analog bandwidth of the sensor is equal to $2.7 \mathrm{GHz}$. The sensor input capacitance is equal to $30 \mathrm{fF}$. The time resolution is about 100 ps and the time measurement window is $180 \mathrm{~ns}$. The imperfections of the sensor which affect the sensor response are compensated by a preliminary calibration. Due to the random errors provided by the measurement equipments and external noise, the measurement repeatability is estimated to $\pm 3 \mathrm{mV}$.

\section{B. Near-field scan test bench}

The principle of the near-field injection test bench used in this study is described in Fig. 12. It relies on a near-field scan table in order to control precisely the position of the near-field probe compared to the device under test. The injection probe can be excited by two types of disturbance sources: harmonic or pulse. Although EM attacks are usually based on pulse disturbances, harmonic disturbances will be used for frequency characterization purpose. They are produced by a RF synthesizer followed by a $50 \mathrm{~W}$ power amplifier. The control of the position of the near-field probe is based on a laser telemeter which constructs the altitude profile of the device under test (DUT). After a proper calibration of the probe position compared to the laser telemeter, the scan altitude $\mathrm{h}_{\text {scan }}$ and probe position are known with a good accuracy. Depending on the telemeter resolution and calibration methodology, the $\mathrm{XY}$ position and scan altitude are known at $+/-100 \mu \mathrm{m}$ and $+/-50$ $\mu \mathrm{m}$ respectively.

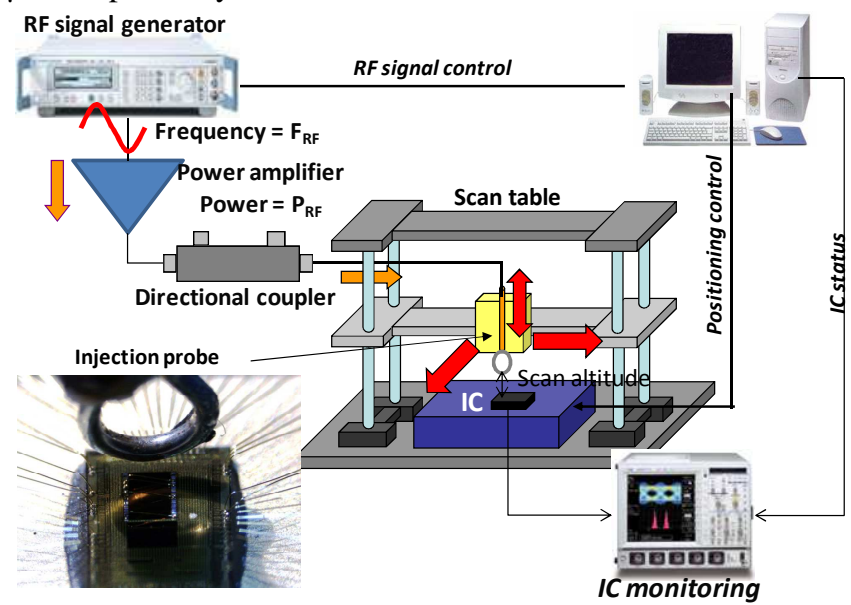

Fig. 12. Near-field injection test bench

\section{EXPERIMENTAL RESULTS}

\section{A. Coupling on $50 \Omega$ microstrip lines}

The first injection tests are performed on the lines of Struct1. The first experiment consists in verifying if a local coupling exists between the injection probe and the lines of Struct1. Fig. 13 presents the evolution of the voltage measured by the sensor on line $0 \mu \mathrm{m}$ according to the probe position. The result is given in the form of a two dimension map. The color of each point of the map gives the voltage measured by sensor when the injection probe is placed above this point. The scan altitude $h_{\text {scan }}$ is $400 \mu \mathrm{m}$ and the $\mathrm{H}$ field probe is oriented along $\mathrm{x}$ axis. The injection frequency and the forward power are set to $400 \mathrm{MHz}$ 
and $20 \mathrm{~W}$ respectively.

The map shows three distinct areas where the induced voltage reaches maxima about some hundreds of millivolts. Two regions are placed above the bonding wires associated to the voltage reference Vref, which bias Struct1, and the sensor output and power supply. The third region is just above Struct1 lines. This result proves that the near-field injection is directly done on Struct1. The measured voltage is induced by the coupling of the tangential component of the magnetic field produced by the injection probe. An obvious method to verify this assertion is to turn the probe by $90^{\circ}$. The amplitude of the voltage measured by sensors of Struct1 is dramatically reduced, as shown in [18].

The voltages measured by the sensors placed on the other lines of Struct1 are also acquired during the near-field injection scan. Fig. 14 presents the evolution of the voltages measured by the sensors placed on four different lines according to the probe position along $\mathrm{x}$ axis. The positions of maximum coupling on these four lines are marked by arrows on Fig. 14. The sensors have not detected these maxima for the same probe positions. Actually, they are shifted along the probe position in accordance with their geometrical separation. This experiment shows that near-field injection can be distinguished between two lines separated by more than $100 \mu \mathrm{m}$. The coupled voltage decreases also rapidly with the scan altitude ${ }_{\text {hscan }}$, as shown in Fig. 15.

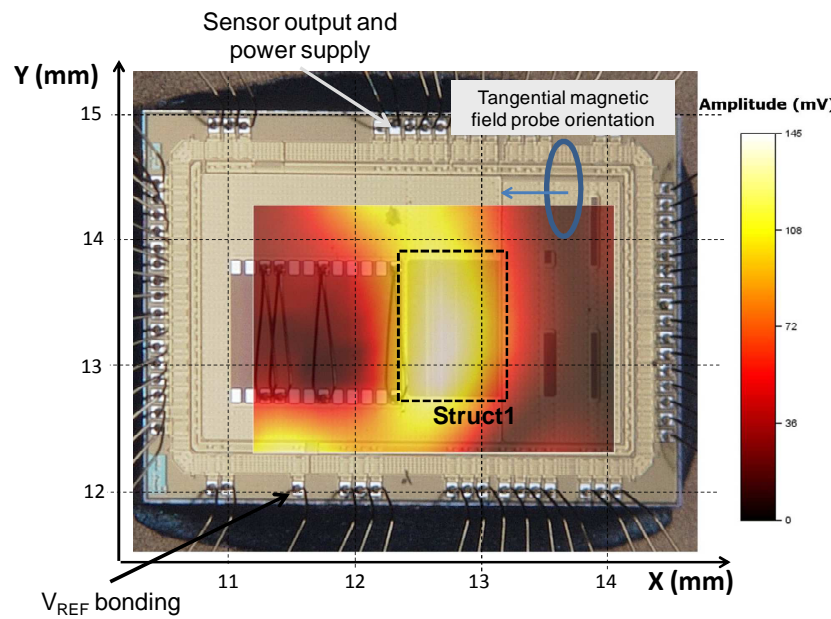

Fig. 13. Coupled voltage on Struct 1 interconnects (line 0 ) according to the probe position $\left(\mathrm{h}_{\mathrm{scan}}=400 \mu \mathrm{m}, \mathrm{F}_{\mathrm{RF}}=400 \mathrm{MHz}, \mathrm{P}_{\mathrm{RF}}=20 \mathrm{~W}\right)$

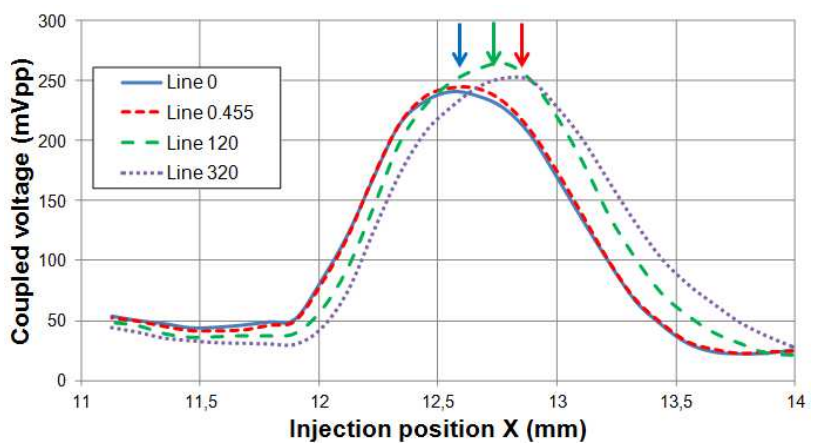

Fig. 14. Evolution of the voltage coupled on four lines of Structl according to the probe position $\left(\mathrm{h}_{\mathrm{scan}}=400 \mu \mathrm{m}, \mathrm{F}_{\mathrm{RF}}=400 \mathrm{MHz}, \mathrm{P}_{\mathrm{RF}}=20 \mathrm{~W}\right)$

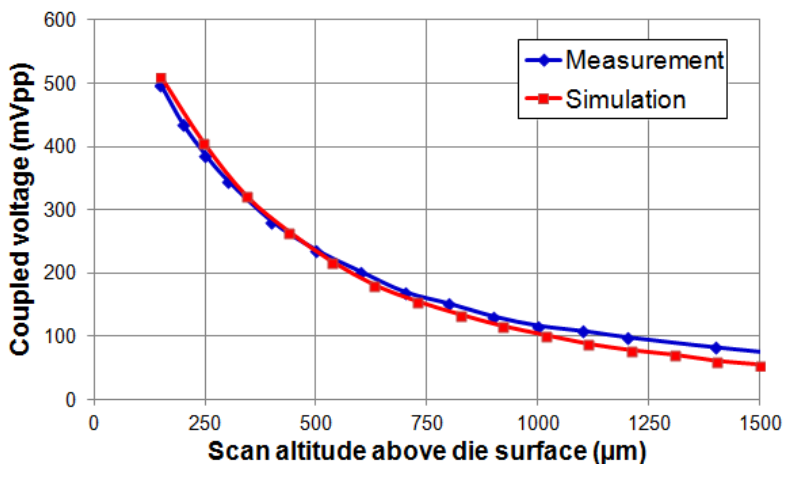

Fig. 15. Coupled voltage vs. scan altitude $\left(F_{R F}=400 \mathrm{MHz}, P_{R F}=20 \mathrm{~W}\right)$

A frequency domain characterization of the coupling is also performed (Fig. 16). The probe is placed above Struct1. The excitation signal frequency is swept from $10 \mathrm{MHz}$ up to 2.5 $\mathrm{GHz}$ and its power is set to $20 \mathrm{~W}$. The coupled voltage tends to increase at $+20 \mathrm{~dB} / \mathrm{dec}$ up to several tens of $\mathrm{MHz}$. Then the coupling increases at a rate close to $+10 \mathrm{~dB} / \mathrm{dec}$. This result indicates that the magnetic field is coupled to a lossy line. It will be confirmed by simulation in the next part.

\section{B. Coupling on digital lines with metal shielding}

The previous experiments are repeated on Struct2. Fig. 17 presents the evolution of the voltage measured by a sensor connected on a digital line of Struct 2 according to the probe position. The scan altitude is $400 \mu \mathrm{m}$ and the $\mathrm{H}$ field probe is oriented along $\mathrm{x}$ axis. The injection frequency and the forward power are set to $400 \mathrm{MHz}$ and $20 \mathrm{~W}$ respectively. The induced voltage is due to the coupling of the tangential component of the magnetic field produced by the injection probe. The induced voltage is ten times weaker than the voltage induced on Struct1 lines. The scan shows two distinct coupling areas: the first one is above the sensor output and power supply bonding, while the other is directly above Struct2. The same voltage is measured by all the sensors on Struct2 interconnects since their spacing is less than the probe resolution.

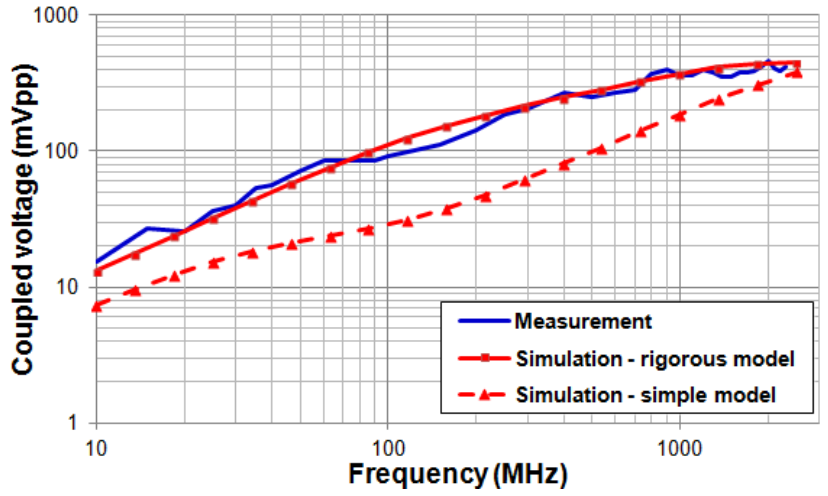

Fig. 16. Measured and simulated evolution of the coupling on the lines Struct1 vs. frequency 


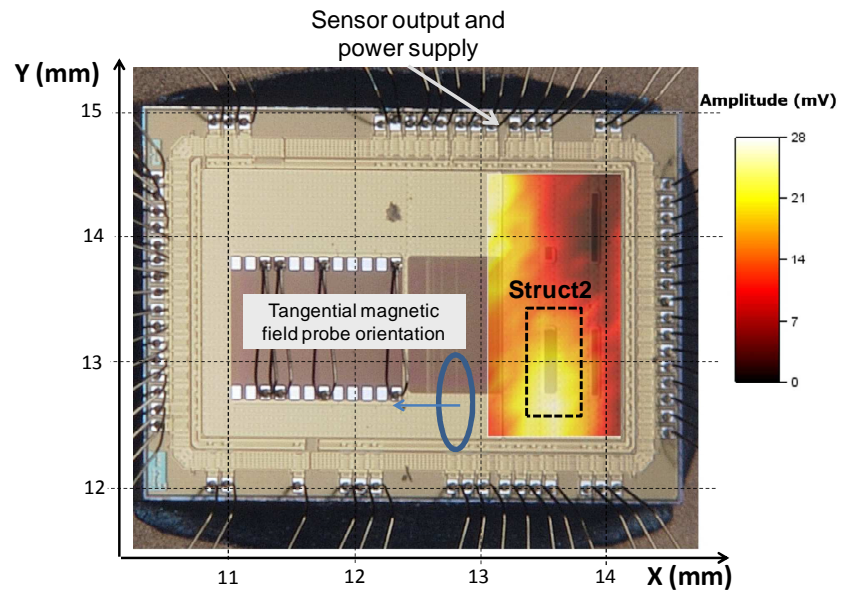

Fig. 17. Coupled voltage on Struct 2 interconnects according to the probe position $\left(\mathrm{h}_{\mathrm{scan}}=400 \mu \mathrm{m}, \mathrm{F}_{\mathrm{RF}}=400 \mathrm{MHz}, \mathrm{P}_{\mathrm{RF}}=20 \mathrm{~W}\right)$

Fig. 18 presents the evolution of the coupled voltage in frequency domain. The excitation signal frequency is swept from $10 \mathrm{MHz}$ up to $2 \mathrm{GHz}$ and its power is set to $20 \mathrm{~W}$. The induced voltage is nearly constant above some tens of $\mathrm{MHz}$ up to $2 \mathrm{GHz}$. This result show that the top level metal shielding reduces the magnetic field coupling without a complete cancellation. In the next part, simulation will help us to clarify the influence of the shielding on the coupling.

\section{SiMULATION RESUlTS}

The modeling flow presented in Fig. 2 is used to simulate the voltage induced on Struct1 and Struct 2 lines by the $\mathrm{H}$ field probe. The methods presented in III.B and III.C are used to model the IC lines and the magnetic field produced by the probe in the vicinity of the IC lines.

\section{A. Modeling of the coupling on $50 \Omega$ microstrip lines}

The microstrip lines designed in Struct1 are actually quite complex. They are formed by several conductors: the traces in metal 3 layer, the reference plane in metal 2 layer and the substrate. The tangential magnetic field is mainly coupled on the equivalent line formed by the reference plane and the substrate, because of its larger surface. This line can be considered as a MIS microstrip line, as shown in Fig. 3. Its equivalent electrical representation is extracted according to the method presented in part III.B. This line is loaded at each end by the bandgap voltage reference and by the sensors connected at the end of each metal 3 traces. The impedance of these loads is extracted from the circuit design and post-layout extraction. The bandgap output is equivalent to a parallel $R C$ circuit with $R=15 \mathrm{k} \Omega$ and $C=70 \mathrm{fF}$. The dimensions and electrical characteristics of each circuit layer are provided by the circuit manufacturer.

The rigorous method presented in part III.C is used to compute the tangential magnetic field produced by the injection probe between the reference plane and the substrate bottom between $10 \mathrm{MHz}$ and $2.5 \mathrm{GHz}$. The voltage induced at the line terminal is finally computed according to the Taylor model and the actual propagation modes determined with the method presented in part III.B. In order to highlight the influence of the multilayered substrate on the magnetic field coupling on the interconnect, the induced voltage is also computed with a simple model: the substrate is considered as a unique insulating layer and the propagation mode along the interconnect is supposed to be quasi-TEM whatever the frequency.

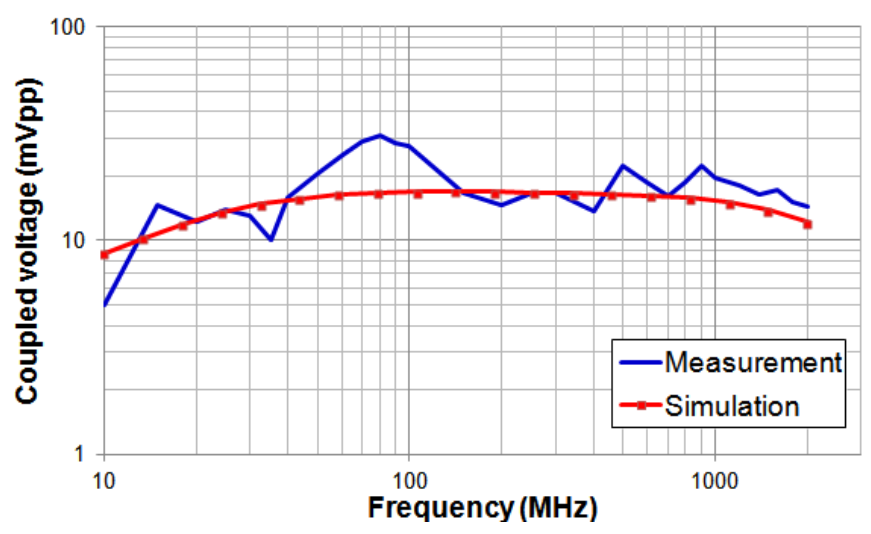

Fig. 18. Measured and simulated evolution of the coupling on a digital line of Struct 2 vs. frequency

Fig. 16 presents the comparison between the measured and simulated evolutions of the voltage coupled on a line of Struct1 according to the frequency. The measurements and simulations with the rigorous model are in good accordance, contrary to the simulations with the simple model which underestimate the coupled voltage up to $2 \mathrm{GHz}$ and do not predict the actual coupling trend with frequency. The induced voltage range and the frequency trend are determined correctly. The substrate resistivity has a strong influence in the increasing rate of the coupled voltage. Above $100 \mathrm{MHz}$, the coupled voltage increases at nearly $10 \mathrm{~dB} / \mathrm{dec}$ because of the slow-wave propagation along the line, which attenuates the induced signal. Moreover, above some hundreds of $\mathrm{MHz}$, the magnetic field penetration within the substrate is attenuated due to skin effect. It should be noted that the coupled voltage stops increasing above $2 \mathrm{GHz}$ because the analog bandwidth of the sensor is limited to $2.7 \mathrm{GHz}$. Fig. 19 presents the influence of the substrate on the coupled voltage. The result shows clearly that a low resistivity substrate (e.g. $0.01 \Omega . \mathrm{cm}$ ) limits the coupled voltage above $100 \mathrm{MHz}$ compared to a high resistive substrate (e.g. $100 \Omega . c m$ ) since a low resistive substrate attenuates both the penetration of the magnetic field within the substrate and the propagation of the signal induced on the interconnect. The coupled voltage depends also on the interconnect characteristics, such as propagation constant and characteristic impedance, and terminal impedances. In this particular case, it explains why the coupled voltage is slightly larger for a substrate resistivity equal to $1 \Omega . \mathrm{cm}$ than for $100 \Omega . \mathrm{cm}$.

In order to validate our model, a second simulation is performed to predict the influence of the scan altitude. Fig. 15 shows the comparison between the measured and simulated induced voltage on lines of Struct1 at $400 \mathrm{MHz}$. A good agreement is observed at short distance to the die. At higher altitude, the injection is less local. The coupling is also 
associated to nearby bonding wires, which is not taken into account in our model.

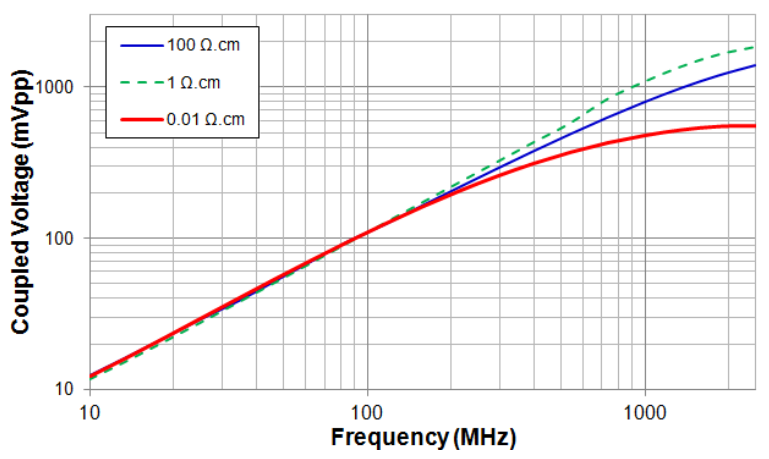

Fig. 19. Effect of the substrate conductivity on the coupled voltage on line of Struct1

\section{B. Modeling of the shielding effect}

The lines of Struct 2 are quite similar to Struct1 lines, except the terminal loads and the presence of a metal shielding on top layer level. This layer is modeled as a conductive layer with the characteristics given by the IC manufacturer. The line formed by the reference plane and the substrate is loaded by the bandgap reference voltage at one end, and by sensors and line driver input at the other end. Fig. 18 presents the comparison between the measured and simulated evolution of the voltage coupled on a line of Struct 2 according to the injection frequency. The simulation reproduces the evolution of the coupled voltage with frequency. The shielding to magnetic field due to the top level metal layer is very weak below $10 \mathrm{MHz}$ and the induced voltage tends to increase. Between $10 \mathrm{MHz}$ and 1 $\mathrm{GHz}$, the coupled voltage is nearly constant. The shielding to magnetic field tends to increase and compensate the magnetic field coupling of the line. The shielding is mainly due to the reflection losses up to $1 \mathrm{GHz}$. Absorption losses becomes significant above $1 \mathrm{GHz}$.

\section{CONCLUSION AND PERSPECTIVES}

Near-field injection at die level is a promising application for various areas such as the analyses of IC susceptibility to electromagnetic interferences and security of cryptographic devices. The development of this method requires accurate models of the physical interaction between the injection probe and the die under test, in order to predict the actual performances of injection probes, evaluate the sensitivity of a circuit to electromagnetic interferences or the threat of electromagnetic attacks.

This paper has presented a first attempt to simulate the voltage induced on integrated circuit interconnects by a magnetic field probe. This problem can be solved by an analysis of the field-to-line coupling. However, due to the multilayer nature of the die and the presence of lossy materials, the propagation along circuit interconnects and the penetration of the magnetic field within the circuit have to be carefully evaluated. The proposed modeling method is based on the theoretical analysis of a parallel plate waveguide to predict the propagation modes according to circuit characteristics. Analytical formulations of the magnetic field dyadic Green's function have been derived to compute the magnetic field produced by an arbitrary oriented injection probe placed in close proximity to a general multilayer circuit.

A test chip with various types of interconnects terminated by on-chip voltage sensors has been developed to measure the voltage induced at die level during near-field injection. The experimental results showed that miniature injection probes placed at short distance to a die may produce voltage fluctuations on circuit interconnects locally. The spatial resolution may reach some hundreds of $\mu \mathrm{m}$ depending on probe dimensions and scan altitude. The induced voltage depends not only on probe dimension, position and excitation, but also on interconnect characteristics, substrate resistivity and top level metallization.

The simulation results are in accordance with near-field injection measurements done with a miniature one turn magnetic field probe. The modeling method can be extended to more complex probe design, e.g. multiturn probe with magnetic core. An extension to time domain has a more practical interest for the evaluation of the threats of pulse injection on secured circuits.

\section{REFERENCES}

[1] S. Zaky, K.G. Balmain, G.R. Dubois, "Susceptibility Mapping”, in Proc. Int. Symp. on EMC, 1992, pp. 439-442.

[2] O. Kroning, M. Krause, M. Leone, "Near field-Immunity Scan on Printed Circuit Board Level", in Proc. SPI, 2010, pp. 101-102.

[3] A. Boyer, E. Sicard, S. Bendhia, « Characterization of the Electromagnetic Susceptibility of Integrated Circuits using a Near Field Scan », Electronic Letters, vol. 43, no. 1, pp. 15-16, 4th Jan. 2007.

[4] T. Dubois, S. Jarrix, A. Penarier, P. Nouvel, D. Gasquet, L. Chusseau, B. Azaïs, " Near-Field Electromagnetic Characterization and Perturbation of Logic Circuits", IEEE Trans. on Instrumentation and Measurement, vol. 57, no. 11, pp. 2398 - 2404, Nov.2008.

[5] D. Castagnet, A. Meresse, and G. Duchamp, "IC's electromagnetic susceptibility: Comparison between a near-field injection method and a direct injection method," in Proc. 3rd Int. Conf. Near-Field Characterization Imag., St. Louis, MO, pp. 296-301, 2007.

[6] T. Dubois, J.J. Laurin, J. Raoult, S. Jarrix, "Effect of Low and High Power Continuous Wave Electromagnetic Interference on a Microwave Oscillator System: From VCO to PLL to QPSK Receiver", IEEE Trans. on EMC, vol. 56, no. 2, pp. 286-293, April 2014.

[7] F. Lafon, F. De-Daran, J. Dupois, "Near field immunity cartography method to characterize IC to fields radiated by an ESD", ICONIC, Barcelona, Spain, Jun. 8-10, 2005.

[8] N. Lacrampe, A. Boyer, B. Vrignon, "Investigation of the indirect effects of VF-TLP ESD pulse injected into a printed circuit board", EMC Europe 2006, Barcelona, Spain, 2006.

[9] G. Muchaidze, J. Koo, Q. Cai, T. Li, L. Han, A. Martwick, K. Wang, J. Min, J. L. Drewniak, D. Pommerenke, "Susceptibility Scanning as a Failure Analysis Tool for System-Level Electrostatic Discharge (ESD) Problems", IEEE Trans. on EMC, vol. 50, no. 2, pp. 268-276, May 2008.

[10] J.M. Schmidt, M. Hutter, "Optical and EM Fault - Attacks on CRTbased RSA: Concrete Results", 15th Austrian Workshop on Microelectronics, 11 Oct. 2007, Graz, Austria. 
[11] S. Ordas, L. Guillaume-Sage, K. Tobich, J. M. Dutertre, P. Maurine, "Evidence of a Larger EM-induced Fault Model", $13^{\text {th }}$ Smart Card Research and Advanced Application Conference (CARDIS2014), Paris, France, Nov. 2014.

[12] F. Poucheret, K. Tobich, M. Lisart, L. Chusseau, B. Robisson, P. Maurine, "Local and Direct EM Injection of Power into CMOS Integrated Circuits", Workshop on Fault Diagnosis and Tolerance in Cryptography, Nara, Japan, Sept. 2011.

[13] P. Bayon, L. Bossuet, A. Aubert, V. Fischer, F. Poucheret, B. Robisson, P. Maurine, "Contactless Electromagnetic Active Attack on Ring Oscillator Based True Random Number Generator", COSADE'2011, Darmstadt, Germany, Feb. 2011.

[14] A. Dehbaoui, J. M. Dutertre, B. Robisson, P. Orsatelli, P. Maurine, A. Tria, "Injection of transient faults using electromagnetic pulses - practical results on a cryptographic system", IACR Cryptology ePrint Archive, no 123, 2012.

[15] A. Boyer, S. Bendhia, E. Sicard, "Modelling of a Mixed-Signal Processor Susceptibility to Near-Field Aggression", IEEE Symp. on EMC, Hawaii, 6 -13 July 2007.

[16] C. Leseigneur, P. Fernandez Lopez, C. Arcambal, D. Baudry, " Near-field coupling model between electronic systems and a transmission line", in Proc. IEEE Int. Symp. on EMC, pp. 22-27, 2010.

[17] H. Hasegawa, M. Furukawa, H. Yanai, "Properties of Microstrip Line on Si-SiO2 System", IEEE Trans. on Microwave Theory and Techniques, vol. MTT-19, no 11, pp. 869 - 881, Nov. 1971.

[18] A. Boyer, B. Vrignon, J. Shepherd, M. Cavarroc, "Evaluation of the Near-Field Injection Method at Integrated Circuit Level", in Proc. EMC Europe 2014, Goteborg, Sweden, pp. 85-90, Sep 1st-4th 2014.

[19] D. Pommerenke, G. Muchaidze, J. Koo, Q. Cai, j. Min, "Application and Limits of IC and PCB Scanning Methods for Immunity Analysis", 18th Int. Zurich Symp. on EMC, 2007.

[20] J. D. Dyson, " Measurement of Near Fields of Antennas and Scatterers", IEEE Trans. on Antenna and Propagation, vol. AP-21, no. 4, pp. $446-$ 460, July 1973.

[21] D. Brooks, "Fusing Current - When Traces Melt Without a Trace", Printed Circuit Design, a Miller Freeman publication, Dec.1998.

[22] M. Kanda, "Standard Probes for Electromagnetic Field Measurements", IEEE Trans on Antennas and Propagation, vol. 41, no. 10, pp. 1349 1364, Oct. 1993.

[23] R. Lundin, "A Handbook Formula for the Inductance of a Single-Layer Circular Coil", in Proc. IEEE, vol. 73, no. 9, pp. 1428-1429, Sep 1985.

[24] R.G. Medhurst, "H.F. Resistance and Self-Capacitance of Single-Layer Solenoids - Part II", Wireless Engineer, no. 24, pp. 80-92, Mar 1947.

[25] C. D. Taylor, R. S. Satterwhite, W. J. Harrison, "The response of terminated two-wire transmission line excited by a non uniform electromagnetic field", IEEE Trans. on Antennas and Propagation, vol. AP-13, pp. 987-989, 1965.

[26] C. E. Baum, T. K Liu, and F. M. Tesche, "On the analysis of general multiconductor transmission line networks," Kirtland AFB, Albuquerque, NM, Interaction Note 350, 1978.

[27] G. Wang, R. W. Dutton, C. S. Rafferty, "Device-Level Simulation of Wave Propagation along Metal-Insulator Semiconductor Interconnects", IEEE Trans. on Microwave Theory and Techniques, vol. 50, no. 4, pp. 1127 - 1136, Apr 2002.

[28] T. G. Livernois, P. B. Katehi, "A Generalized Method for Deriving Space-Domain Green's Function in a Shielded, Multilayer Substrate Structure with Applications to MIS Slow-Wave Transmission Lines", IEEE Trans on Microwave Theory and Techniques, vol. 37, no 11, pp. 1761 - 1767, Nov 1989.

[29] A. Weisshaar, H. Lan, A. Luoh, "Accurate Closed-Form Expresssions for the Frequency-Dependent Line Parameters of the On-Chip Interconnects on Lossy Silicon Substrate", IEEE Trans. on Advanced Packaging, vol. 25, no 2, pp. 288 - 296, May 2002.

[30] H. Hasegawa, "Analysis of Interconnection Delay on Very High-Speed LSI/VLSI Chips Using MIS Microstrip Line Model", IEEE Trans. on Microwave Theory and Techniques, vol. MTT-32, no 12, pp. 1721 1727, Dec. 1984.

[31] Y. Leonard Chow, "An Approximate Dynamic Green's Function in Three Dimensions for Finite Length Microstripline", IEEE Trans. on Microwave Theory and Techniques, vol. MTT-28, no 4, pp. 393 - 397, Apr. 1980.
[32] L. Vegni, R. Cicchetti, P. Capece, "Spectral Dyadic Green's Function Formulation for Planar Integrated Structures", IEEE Trans on Antennas and Propagation, vol. 36, no 8, pp. 1057 - 1065, Aug. 1988.

[33] N. K. Das, D. M. Pozar, "A Generalized Spectral-Domain Green's Function for Multilayer Dielectric Substrates with Applications to Multilayer Transmission Lines", IEEE Trans. on Microwave Theory and Techniques, vol. MTT-35, no. 3, pp. 326 - 335, Mar 1987.

[34] G. Dural, M. I. Aksun, "Closed-Form Green's Functions for General Sources and Stratified Media", IEEE Trans. on Microwave Theory and Techniques, vol. 43, no 7, pp. 1545 - 1552, Jul. 1995.

[35] P. R. Bannister, "Applications of Complex Image Theory", Radio Science, vol. 21, no. 4, pp 605-616, Jul. - Aug. 1986.

[36] M. I. Aksun, G. Dural, "Clarification of Issues on the Closed-Form Green's Functions in Stratified Media", IEEE Trans. on Antennas and Propagation, vol. 53, no 11, pp. 3644 - 3653, Nov. 2005.

[37] FEKO, Electromagnetic Simulation Software, more information on www.feko.info, 2015.

[38] S. Ben Dhia, E. Sicard, F. Caignet, "A new method for measuring signal integrity in CMOS ICs", Microelectronic Int. Journal, vol. 17, no. 1, pp. 17-21, Jan. 2000.

[39] S. Ben Dhia, A. Boyer, B. Vrignon, M. Deobarro, T. V. Dinh, "On-Chip Noise Sensor for Integrated Circuit Susceptibility Investigations", IEEE Trans. on Instrumentation and Measurement, vol. 61, no. 3, pp. 696-707, Mar. 2012.

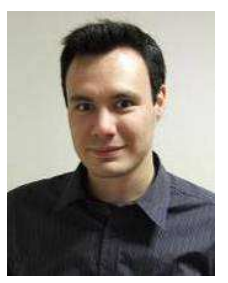

Alexandre Boyer obtained a Masters degree in electrical engineering in 2004 and a $\mathrm{PhD}$ in Electronics from the Institut Nationale des Sciences Appliquées (INSA) in Toulouse, France, in 2007. He is currently an Associate Professor in the Department of Electrical and Computer Engineering at INSA, Toulouse. He is leading his research at the Laboratoire d'Analyse et d'Architecture des Systèmes (LAAS-CNRS), as part of the 'Energy and Embedded Systems' research group. His current research interests include IC susceptibility and reliability modeling, and computer aided design (CAD) tool development for EMC (IC-EMC freeware).

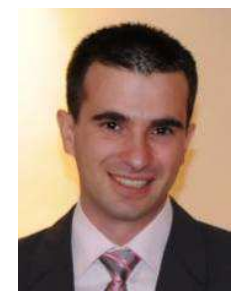

Bertrand Vrignon was born in Tours, France, in 1979. He received his engineering diploma from the Ecole Supérieure d'Electronique de l'Ouest, Angers, France, in 2002, and a Ph.D. in electronic design from the National Institute of Applied Sciences, Toulouse, France, in 2005. He was with STMicroelectronics, Crolles, where he was engaged in the characterization of low electromagnetic emission guidelines for ICs. Since 2005, he has been a Research Engineer with Freescale Semiconductors, Toulouse, where he is engaged in electromagnetic compatibility studies at IC level. His current research interests include several aspects of design methodology to reduce emission, and improve noise susceptibility of deep-submicron ICs.

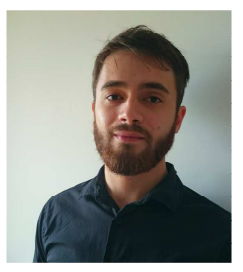

Manuel Cavarroc obtained a Bachelor of Science specialized in tests and instrumentation in complex environments, from the University Institute of Technology Paul Sabatier in Toulouse (France). Since 2013, he worked at LAAS-CNRS as an assistant engineer in the Instrumentation Design and Characterization (I2C) service. $\mathrm{He}$ provides a technical support for the research projects of Energy and Embedded Systems group, inter alia, he is engaged to design and automate test benches to realize EMC measurements and component characterization. 